\title{
Crystal Grain Observation Using a Segmented Backscattered Electron Detector
}

\author{
Takeshi Otsuka ${ }^{1}$, Masaya Hara ${ }^{1}$, Natasha Erdman $^{2}$ and Shin-ichi Kitamura ${ }^{1}$ \\ 1. JEOL Ltd., Akishima, Tokyo, Japan \\ 2. JEOL USA, Inc., Peabody, MA, USA
}

Analyzing grain size in metallic specimens is important for quality-control of metal alloys. In the case of a scanning electron microscope (SEM), an electron backscatter diffraction (EBSD) system is generally used for grain structure analysis. In EBSD, the crystal orientation is determined by analyzing Kikuchi bands with an electron beam scanned over a tilted specimen. The electron beam is scanned on the specimen and a crystal orientation map, known as inverse pole figure (IPF) map, can be obtained. The grain sizes are determined by the IPF map.

During routine SEM observation of a non-tilted specimen, the Kikuchi bands are also projected on a backscattered electron detector (BED) which located in front of the specimen. If BED is segmented, signal differences due to Kikuchi bands will be detected among the segments. When the crystal orientation of the specimen is changed, the Kikuchi bands and the signal differences are also changed. In this study, we tried to measure the signal differences of segmented BED during electron beam scanning, and obtained the grain distribution.

The experimental system is shown in Fig. 1. The semiconductor detector with 16 azimuthal divisions was used as the segmented BED which was located under the objective lens. The specimen was a flat polycrystalline gold which was placed horizontally. Electron beam energy was $5 \mathrm{keV} . I_{i}(\mathrm{i}=1 \sim 16)$ is signal intensity from the i-th segmented BED. For clarifying the signal difference, we define the brightness as $d=\sqrt{\sum_{i}\left(I_{i}-I_{0_{i}}\right)^{2}}$, where $I_{0_{i}}$ is a reference of signal intensity pattern. A new scanning image (as a recognition image) which has crystal information can be displayed using $d$. If the signal intensity pattern $I$ is similar to the reference $I_{0}$, it is displayed in black, which means that, the black region in the recognition image shows the same crystal orientation.

A recognition image taken by this system is shown in Fig. 2(a). Measurement time of this image is only about $1 \mathrm{~min}$. The reference point is the center of the image. Therefore, the area at the center is displayed in black. A compositional image of the same area is shown in Fig. 2(b). In comparison between (a) and (b), the boundary (arrow in Fig. 2(b)) is not visible. On the other hand, another boundary in Fig. 2(b) (arrow head) is clearer than that in Fig. 2(a). This method is not perfect to distinguish the difference between crystal grains which are not similar to the reference. Therefore, we increased reference points and defined RGB brightness as below

$d_{R}=\sqrt{\sum_{i}\left(I_{i}-I_{R i}\right)^{2}}, d_{G}=\sqrt{\sum_{i}\left(I_{i}-I_{G i}\right)^{2}}, d_{B}=\sqrt{\sum_{i}\left(I_{i}-I_{B i}\right)^{2}}$, which shows color R, G and B,

respectively (Fig. 3(a), color recognition image). Where $I_{R i}, I_{G i}$ and $I_{B i}$ are reference points. By this method, crystal grains which are difficult to distinguish (Fig. 2(a)) can be identified in the image of Fig. 3 (a). An IPF map of the same area was taken by EBSD system (Fig. 3(b)). The same color areas (* and \#) in Fig. 3(a) have the same orientation in the IPF map. This confirms that the areas in the same color in Fig. 3(a) have the same orientation. 
This technology does not need sample tilt and long acquisition time in comparison with EBSD. For example, it is applicable to crystal grain observation while capturing SEM image because the specimen is set horizontally, or a specimen can be under heating/cooling and tensile stress because measurement time is fast.

(a)

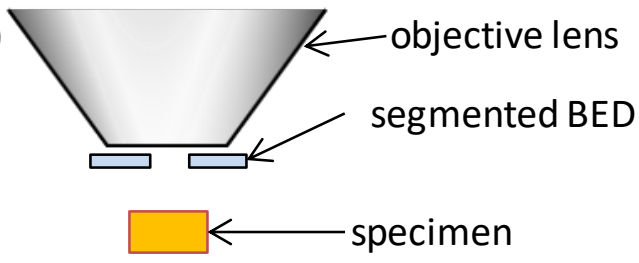

(b)

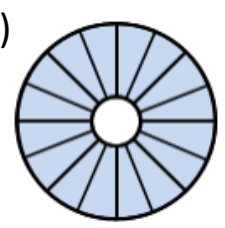

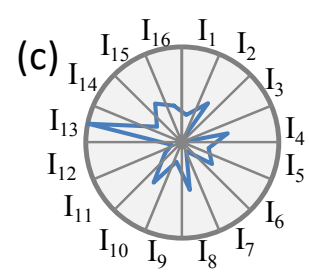

Figure 1. Schematic diagram of (a) the system and (b) shape of segmented BED. (c) Signal intensity pattern of segmented BEI.
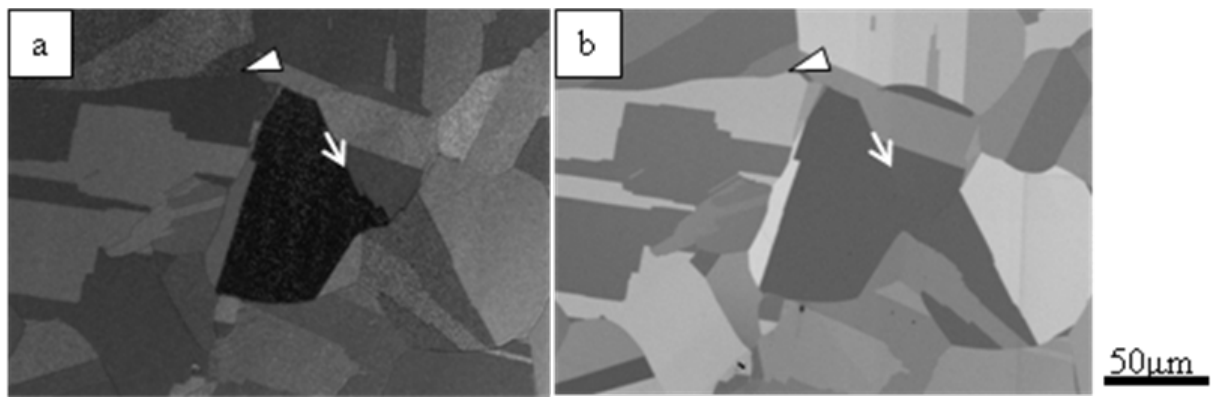

Figure 2. (a) Recognition image. (b) Compositional image (polycrystalline gold, landing energy is 5 $\mathrm{keV}$.)
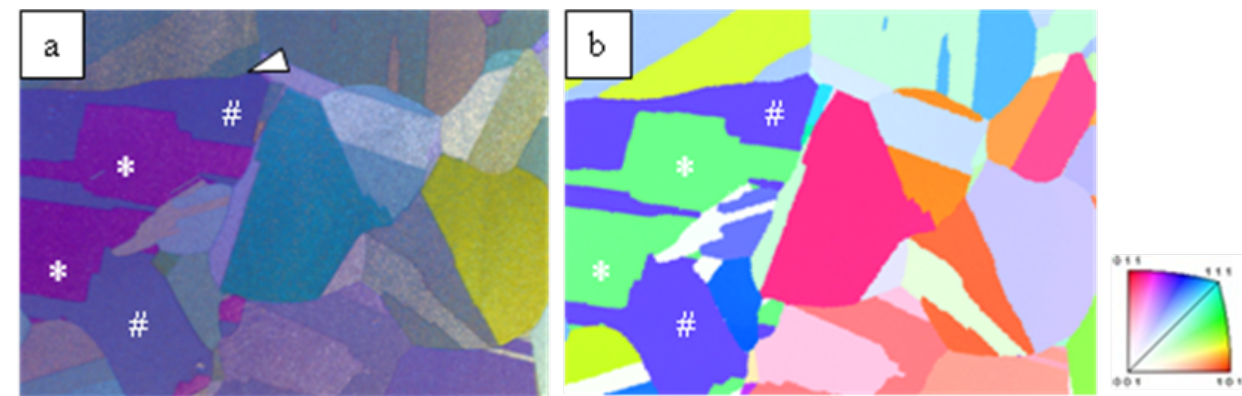

Figure 3. (a) Color recognition image. (b) IPF map taken by EBSD. 